\title{
The efficacy of insecticide-treated window screens and eaves against Anopheles mosquitoes: a scoping review
}

\author{
Beverly I. Anaele ${ }^{1 *}$ (D), Karan Varshney ${ }^{1,2}$, Francis S. O. Ugwu ${ }^{3}$ and Rosemary Frasso ${ }^{1}$
}

\begin{abstract}
Background: Female mosquitoes serve as vectors for a host of illnesses, including malaria, spread by the Plasmodium parasite. Despite monumental strides to reduce this disease burden through tools such as bed nets, the rate of these gains is slowing. Ongoing disruptions related to the COVID-19 pandemic may also negatively impact gains. The following scoping review was conducted to examine novel means of reversing this trend by exploring the efficacy of insecticide-treated window screens or eaves to reduce Anopheles mosquito bites, mosquito house entry, and density.

Methods: Two reviewers independently searched PubMed, Scopus, and ProQuest databases on 10 July, 2020 for peer-reviewed studies using insecticide-treated screens or eaves in malaria-endemic countries. These articles were published in English between the years 2000-2020. Upon collection, the reports were stratified into categories of biting incidence and protective efficacy, mosquito entry and density, and mosquito mortality.

Results: Thirteen out of 2180 articles were included in the final review. Eaves treated with beta-cyfluthrin, transfluthrin or bendiocarb insecticides were found to produce vast drops in blood-feeding, biting or mosquito prevalence. Transfluthrin-treated eaves were reported to have greater efficacy at reducing mosquito biting: Rates dropped by $100 \%$ both indoors and outdoors under eave ribbon treatments of $0.2 \%$ transfluthrin $(95 \% \mathrm{Cl} 0.00-0.00 ; \mathrm{p}<0.001$ ). Additionally, co-treating window screens and eaves with polyacrylate-binding agents and with pirimiphos-methyl has been shown to retain insecticidal potency after several washes, with a mosquito mortality rate of $94 \%$ after 20 washes $(95 \% \mathrm{Cl} 0.74-0.98 ; \mathrm{p}<0.001)$.

Conclusions: The results from this scoping review suggest that there is value in implementing treated eave tubes or window screens. More data are needed to study the longevity of screens and household attitudes toward these interventions.
\end{abstract}

Keywords: Malaria, Window screen, Eave, Insecticide, Scoping review

\section{Background}

In 2019, the World Health Organization (WHO) reported a total of 229 million cases of malaria [1]. Sub-Saharan Africa carries the majority of the malaria burden, equating to $94 \%$ of worldwide cases. Several reasons for this

\footnotetext{
*Correspondence: beverly.anaele@gmail.com

${ }^{1}$ College of Population Health, Thomas Jefferson University, Philadelphia, PA, USA
}

Full list of author information is available at the end of the article disproportionality exist, one of which lies in housing infrastructure [2]. As evidence of this, a study indicated that on average malaria doubled among children with the lowest socio-economic status (SES) when measured in household wealth, parental education level and job status relative to children in the same community with the highest SES [3]. The researchers hypothesized that this discrepancy in malaria contraction may have derived from differences in access to healthcare, knowledge of

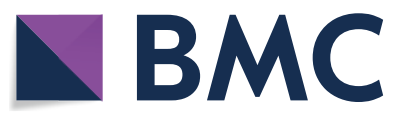

(c) The Author(s) 2021. Open Access This article is licensed under a Creative Commons Attribution 4.0 International License, which permits use, sharing, adaptation, distribution and reproduction in any medium or format, as long as you give appropriate credit to the original author(s) and the source, provide a link to the Creative Commons licence, and indicate if changes were made. The images or other third party material in this article are included in the article's Creative Commons licence, unless indicated otherwise in a credit line to the material. If material is not included in the article's Creative Commons licence and your intended use is not permitted by statutory regulation or exceeds the permitted use, you will need to obtain permission directly from the copyright holder. To view a copy of this licence, visit http://creativecommons.org/licenses/by/4.0/. The Creative Commons Public Domain Dedication waiver (http://creativeco mmons.org/publicdomain/zero/1.0/) applies to the data made available in this article, unless otherwise stated in a credit line to the data. 
treatment opportunities, food security, and housing quality [3].

With improved housing comes a reduced risk of contracting malaria due to lowered exposure to mosquitoes $[2,4,5]$. A 2001-2017 cross-sectional analysis using data from 33 African countries found that improved housing lowered the risk of child mortality from malaria by $12-18 \%$ [4]. Openings in walls and other points of entry, once closed, can bar vectors from biting indoors during their active hours. Tools such as insecticide-treated window screens and eaves are designed to do just that, along with the added community effect of reducing mosquito populations due to the insecticide treatment. However, these devices are not as widely prioritized in the fight against malaria compared to bed net programmes or indoor residual spraying [6]. More concrete research displaying the benefits of window screens and eaves is required [6]. Added research will help to motivate all critical stakeholders, such as policymakers, to invest in said tools. Addressing housing quality could help to alleviate other issues of poverty by identifying household needs and concerns.

The following review addresses the call for more research by synthesizing and summarizing current scientific knowledge of housing interventions to inform policy. More specifically, this scoping review provides a detailed account of the literature, assessing whether chemical insecticide-treated window screens and eaves are capable of reducing malaria incidence, prevalence, and mosquito biting densities within malaria-endemic countries.

\section{Methods \\ Search strategy}

On 10 July, 2020, the research team performed a literature search exploring PubMed, Scopus, and ProQuest databases using the terms found in Table 1. These search terms were selected through collaboration with a research librarian and incorporated medical subject headings and keywords. Both categories pertained to malaria-endemic regions, insecticides or pyrethroids, window screens, and eaves. To address outcomes, search terms on epidemiology, mosquito bites, disease prevalence, and incidence were also included.

\section{Inclusion and exclusion criteria}

The research team incorporated guidelines from the Preferred Reporting Items for Systematic Reviews and Meta-Analyses (PRISMA) study selection process [7]. The inclusion criteria comprised: (1) data reported from malaria-endemic countries in the WHO regions of subSaharan Africa, Southeast Asia, the Eastern Mediterranean, the Western Pacific, and South America; (2) studies reported in English; (3) interventions investigating Anopheles mosquitoes using insecticide-treated netting for window screens and eaves; (4) studies that used empirical methods, meaning the collection and analysis

Table.1 Search strategies and databases performed on July 10, 2020

\begin{tabular}{|c|c|c|}
\hline Database & Search & Hits \\
\hline PubMed & $\begin{array}{l}\text { Sub-Saharan Africa OR "Africa South of the Sahara"[Mesh] OR Southeast Asia OR "Asia, Southeastern"[Mesh] OR Eastern Mediter- } \\
\text { ranean OR"Morocco"[Mesh] OR "Cyprus"[Mesh] OR Western Pacific OR "Ecuador"[Mesh] OR "Peru"[Mesh] OR "Samoa"[Mesh] OR } \\
\text { "Independent State of Samoa"[Mesh] OR"Palau"[Mesh] OR South America OR "South America"[Mesh] AND Malaria OR"Malaria"[MeSH } \\
\text { Terms] OR "Plasmodium"[Mesh] AND insecticide OR insects OR Insecticidal OR "Pyrethrins"[Mesh] OR pyrethroid OR "Mosquito } \\
\text { Control"[Mesh] OR Insecticide-treated AND windows OR window OR screens OR screen OR eaves OR eave AND Mosquito bites OR } \\
\text { Mosquito bite OR"culicidae"[MeSH Terms] OR mosquito OR "bites and stings"[MeSH Terms] OR bites stings OR bites OR "Malaria/ } \\
\text { epidemiology"[Mesh] OR "Malaria/prevention and control"[Mesh] }\end{array}$ & 637 \\
\hline ProQuest & $\begin{array}{l}\text { (Sub-Saharan Africa OR Africa OR Southeast Asia OR SOUTHEAST ASIA OR Mediterranean OR Mediterranean Area OR Morocco } \\
\text { OR Cyprus OR Western Pacific OR Ecuador OR Peru OR Samoa OR Palau OR South America OR SOUTH AMERICA) AND (malaria } \\
\text { OR plasmodium OR plasmodium berghei OR plasmodium chabaudi OR plasmodium cynomolgi OR plasmodium falciparum OR } \\
\text { plasmodium falciparum (malaria parasite) OR plasmodium gallinaceum OR plasmodium knowlesi OR plasmodium malariae OR } \\
\text { plasmodium ovale OR plasmodium relictum OR plasmodium vinckei OR plasmodium vivax OR plasmodium yoelii) AND (Insecticide } \\
\text { OR insecticides OR pyrethrins OR pyrethroid OR mosquito control) AND (window OR windows OR window screens OR screen doors } \\
\text { OR eaves OR eaves.) AND (bites OR bites \& stings OR bites, human) }\end{array}$ & 1018 \\
\hline Scopus & $\begin{array}{l}\text { TITLE-ABS-KEY sub-saharan AND africa OR TITLE-ABS-KEY africa AND south AND of AND the AND sahara OR TITLE-ABS-KEY southeast } \\
\text { AND asia OR TITLE-ABS-KEY eastern AND mediterranean ORTITLE-ABS-KEY morocco OR TITLE-ABS-KEY southeastern AND asia OR } \\
\text { TITLE-ABS-KEY cyprus OR TITLE-ABS-KEY western AND pacific OR TITLE-ABS-KEY ecuador OR TITLE-ABS-KEY peru OR TITLE-ABS- } \\
\text { KEY samoa OR TITLE-ABS-KEY independent AND state AND of AND samoa OR TITLE-ABS-KEY palau OR TITLE-ABS-KEY south AND } \\
\text { america AND TITLE-ABS-KEY malaria OR TITLE-ABS-KEY plasmodium AND TITLE-ABS-KEY insecticide OR TITLE-ABS-KEY insects OR } \\
\text { TITLE-ABS-KEY insecticidal OR TITLE-ABS-KEY pyrethrins OR TITLE-ABS-KEY pyrethroid OR TITLE-ABS-KEY mosquito AND control OR } \\
\text { TITLE-ABS-KEY insecticide-treated AND TITLE-ABS-KEY windows OR TITLE-ABS-KEY window OR TITLE-ABS-KEY screens OR TITLE-ABS- } \\
\text { KEY screen OR TITLE-ABS-KEY eaves OR TITLE-ABS-KEY eave OR TITLE-ABS-KEY net OR TITLE-ABS-KEY nets OR TITLE-ABS-KEY bednet } \\
\text { OR TITLE-ABS-KEY bednets OR TITLE-ABS-KEY bed AND net OR TITLE-ABS-KEY bed AND nets AND TITLE-ABS-KEY epidemiology OR } \\
\text { TITLE-ABS-KEY mosquito AND bites OR TITLE-ABS-KEY mosquito AND bite OR TITLE-ABS-KEY culicidae OR TITLE-ABS-KEY mosquito } \\
\text { OR TITLE-ABS-KEY stings OR TITLE-ABS-KEY bites OR TITLE-ABS-KEY prevalence OR TITLE-ABS-KEY incidence }\end{array}$ & 525 \\
\hline
\end{tabular}


of data using the scientific method; and (5) studies that were published in the years 2000-2020. Articles that investigated bed nets or curtains alone were excluded from the study.

\section{Data extraction}

Data were collected in RefWorks Legacy, and each article was independently assessed by two reviewers (BIA and KV). Data from each study were qualitatively synthesized into categories of biting incidence and protective efficacy, mosquito entry and density, mosquito mortality, and household member attitudes toward each intervention. The Critical Appraisal Skills Programme Checklist (CASP) for case-control studies was used to evaluate the quality of these reports, where groups exposed to the insecticide were taken as cases and non-exposed groups were categorized as controls [8]. Randomized controlled trials (RCTs) were appraised with the CASP for RCT studies [9]. Reviewers discussed and resolved any discrepancies.

\section{Results}

Out of 2180 results, 282 duplicates were removed and 1834 studies excluded after assessing their titles and abstracts. Thirteen final articles were identified out of the remaining 64 studies that met all the inclusion criteria (Fig. 1) [10-22]. These 13 articles assessed interventions based on their overall effect on biting incidence, protective efficacy, mosquito entry and density, mosquito mortality, and participant attitudes toward the mosquito

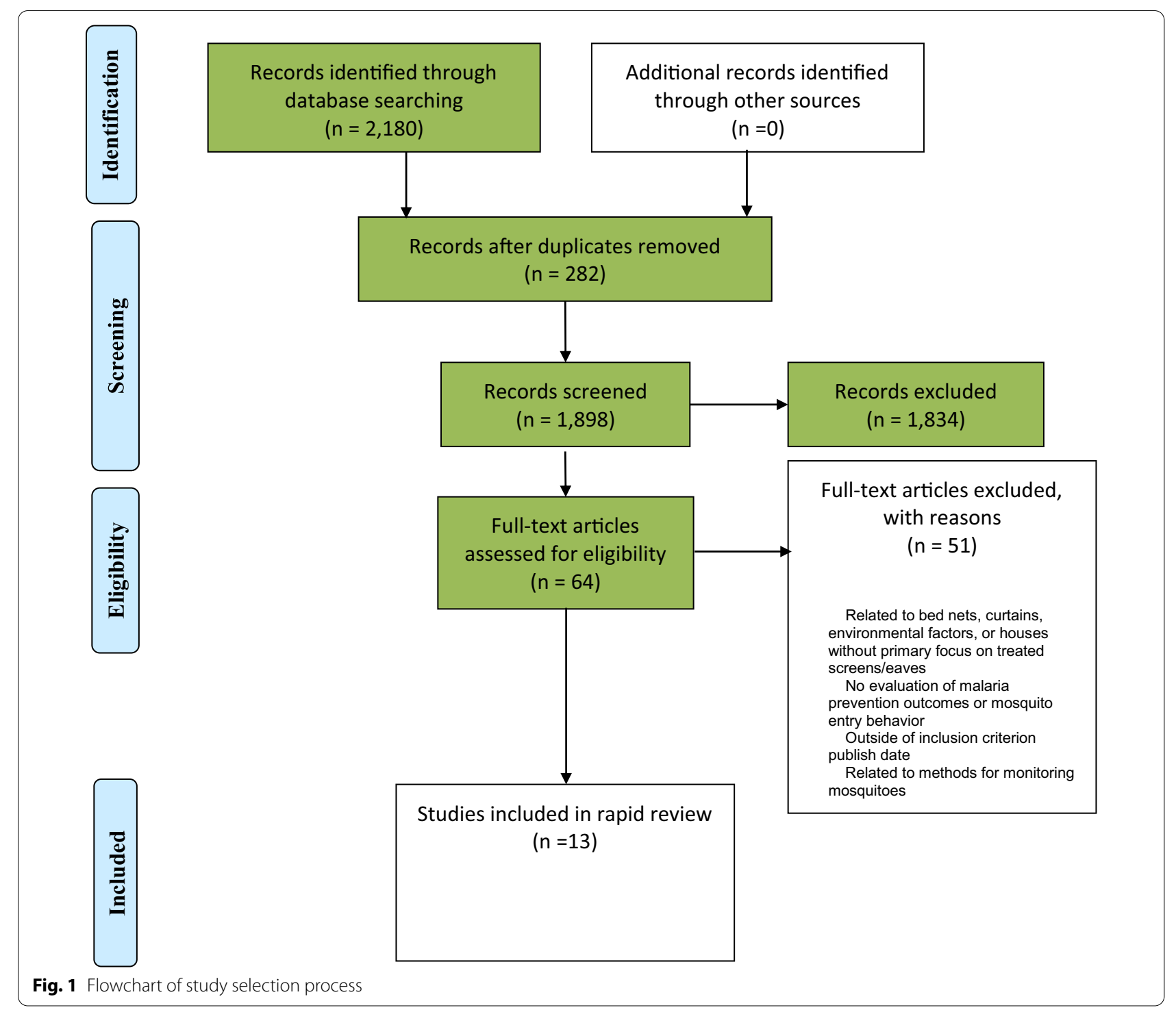


control interventions. All articles were of high quality based on their appropriate appraisal checklist (Table 2).

\section{Characteristics of studies}

Population demographics of all articles varied widely, particularly in the Anopheles species that researchers studied (Table 3). Three interventions utilized RCT study designs and the remaining 10 used case-control releaserecapture, wild mosquito recruitment, and/or experimental hut studies (Table 3). Studies using experimental huts first reconstructed West African-style homes. These homes were modified to include multiple eave tubes at eave level. Other reports identified as village studies, as they incorporated housing units already present in a community. To collect mosquitoes, the overnight releaserecapture method or the recruitment of wild mosquitoes was used. With the release-recapture method, laboratory-reared mosquitoes were released into the experimental enclosure and later captured to record their position and status. The recruitment of wild mosquitoes, conversely, involved the capture of mosquitoes that were not raised in a controlled setting.

Most studies $(n=10)$ reported on treated eave inserts, while three studies investigated both window screens and eaves. All data were collected in African countries: four studies took place in Tanzania, whereas three articles were aggregated in Côte d'Ivoire and three in Kenya. All other studies produced results from Mozambique, Bénin, and Zambia.

All reports $(\mathrm{n}=13)$ indicated that their studies included human volunteers. Of the 13 articles, eight involved adult participants, one involved children, and four did not identify participant ages. Five studies comprised all male volunteers and the eight remaining studies did not provide information on the gender of their volunteers. Insect samples ranged from 90 to 11,362 released or collected mosquitoes. Twelve studies re-evaluated the intervention at least twice, while one design did not clearly state whether treatment replication took place (Table 2).

\section{Biting incidence and protective efficacy}

Five of the 13 studies indicated the intervention effects on mosquito-biting incidence and protective efficacy (Table 4). Three of these reports utilized transfluthrin insecticide against mosquito vectors, of which one study found that eave ribbons treated with insecticide concentrations of $0.2 \%$ led to $100 \%$ reductions in indoor and outdoor biting with zero average nightly mosquito catches (95\% CI 0.00-0.00; p <0.001) [10]. In this report, volunteers assigned to houses with eaves covered in 10 times less transfluthrin $(0.02 \%)$ still experienced $77.2 \%$ protection indoors with three average nightly catches $(95 \%$ CI
$0.32-5.18 ; \mathrm{p}<0.001)$ and $56.2 \%$ protection outdoors with 46 average nightly catches (95\% CI 23.5-68.6; $\mathrm{p}<0.001)$.

Mmbando et al. indicated that eaves treated with transfluthrin afforded $96 \%$ indoor and $84 \%$ outdoor protection against Anopheles arabiensis [10]. Drastically lower, though, were the treatment results on Anopheles funestus, as volunteers experienced only $42 \%$ protection indoors and $40 \%$ outdoor protection [10]. As a form of community defence, one study reported that if $80 \%$ of households installed transfluthrin-eaves, an entire community would experience a state of herd immunity [8].

Mwanga et al. [11] found that eave ribbons treated with transfluthrin protected participants who slept under a net at $83 \%$ efficacy indoors and $62 \%$ outdoors. Conversely, volunteers who did not sleep under a net experienced $57 \%$ protection indoors and $48 \%$ outdoors against An. arabiensis mosquitoes [11]. Volunteers who slept under nets and with treated eaves had constant levels of protection, whereas those who did not sleep under a net experienced increased levels of protection when eave ribbons were added to the mix [11].

\section{Mosquito entry and density}

Seven studies reported the intervention outcomes on mosquito entry and indoor density (Table 4). In Barreaux et al.s [12] report, inserts treated with $10 \%$ betacyfluthrin and placed in eave tubes using the In2Care tool resulted in $0-0.4 \%$ of mosquitoes entering houses with closed windows. This method is known as the Lethal House Lure. The control hut had around $50-80 \%$ of mosquitoes entering the structure when eaves were untreated [12].

Snetselaar et al. [13] reported that directly applying bendiocarb to eaves produced an average recapture rate of $21 \%$ for both Anopheles gambiae s.s. (95\% CI 18-25\%) and An. arabiensis (95\% CI 14-27\%). Similarly, another report found that bendiocarb-eaves resulted in $50-70 \%$ lower recapture rates than their controls [14]. These treated eaves also performed as well as long-lasting insecticidal netting (LLIN) and open eaves together [14]. When Snetselaar et al. [13] experimented with deltamethrin, they found it allowed $18 \%$ more An. gambiae s.s. $(95 \%$ CI $26-51 \%)$ and $1 \%$ more An. arabiensis $(95 \%$ CI 18-25\%) into houses compared to bendiocarb. Microscopically, Gouissi et al. [15] found that covering eaves and windows with Olyset Nets (impregnated with permethrin) reduced Plasmodium parasite density in participant blood by two times compared to the control.

\section{Mosquito mortality}

In addition to outcomes related to mosquito entry behaviour, seven of the included studies generated reports based on mosquito mortality (Table 4). These 
Table.2 Critical appraisal checklist

\begin{tabular}{|c|c|c|c|c|c|c|c|c|c|c|c|c|c|}
\hline $\begin{array}{l}\text { Article } \\
\text { number }\end{array}$ & References & $\# 1$ & \#2 & $\# 3$ & $\# 4$ & $\# 5$ & $\# \mathbf{6 a}$ & $\# 6 \mathbf{b}$ & $\# 7$ & $\# 8$ & $\# 9$ & $\# 10$ & \#11 \\
\hline 1 & $\begin{array}{l}\text { Barreaux et al. } \\
{[19]}\end{array}$ & Yes & Yes & Yes & Yes & Yes & Yes & Yes & $\begin{array}{l}\text { At least six replicates of } \\
\text { treatment }\end{array}$ & Very & Yes & Yes & Yes \\
\hline 2 & $\begin{array}{l}\text { Barreaux et al. } \\
{[12]}\end{array}$ & Yes & Yes & Yes & Yes & Yes & Yes & Yes & $\begin{array}{l}\text { At least ten replicates of } \\
\text { treatment }\end{array}$ & Very & Yes & Yes & Yes \\
\hline 3 & $\begin{array}{l}\text { Chinula et al. } \\
{[17]}\end{array}$ & Yes & Yes & Yes & Yes & Yes & Yes & Yes & $\begin{array}{l}\text { Two replicates of } \\
\text { treatment }\end{array}$ & Very & Yes & Yes & Yes \\
\hline 4 & $\begin{array}{l}\text { Gouissi et al. } \\
{[15]}\end{array}$ & Yes & Yes & Yes & Yes & Yes & Yes & Yes & Yes & Yes & Yes & Yes & Yes \\
\hline 5 & $\begin{array}{l}\text { Kampango et al. } \\
{[20]}\end{array}$ & Yes & Yes & Yes & $\begin{array}{l}\text { Can't } \\
\text { tell }\end{array}$ & Yes & Yes & & Yes & Yes & Yes & Yes & Yes \\
\hline 6 & $\begin{array}{l}\text { Menger et al. } \\
{[21]}\end{array}$ & Yes & Yes & Yes & $\begin{array}{l}\text { Can't } \\
\text { tell }\end{array}$ & Yes & Yes & & Yes & Yes & Yes & Yes & Yes \\
\hline 7 & $\begin{array}{l}\text { Menger et al. } \\
{[22]}\end{array}$ & Yes & Yes & Yes & $\begin{array}{l}\text { Can't } \\
\text { tell }\end{array}$ & Yes & Yes & & Yes & Yes & Yes & Yes & Yes \\
\hline 8 & $\begin{array}{l}\text { Mmbando et al. } \\
{[18]}\end{array}$ & Yes & Yes & Yes & Yes & Yes & Yes & Yes & $\begin{array}{l}\text { At least five replicates } \\
\text { of treatment }\end{array}$ & Yes & Yes & Yes & Yes \\
\hline 9 & $\begin{array}{l}\text { Mmbando et al. } \\
{[10]}\end{array}$ & Yes & Yes & Yes & Yes & Yes & Yes & Yes & $\begin{array}{l}\text { At least } 20 \text { replicates of } \\
\text { treatment }\end{array}$ & Yes & Yes & Yes & Yes \\
\hline 10 & $\begin{array}{l}\text { Mwanga et al. } \\
\text { [11] }\end{array}$ & Yes & Yes & Yes & Yes & Yes & Yes & Yes & $\begin{array}{l}\text { At least } 25 \text { replicates of } \\
\text { treatment (for protective } \\
\text { efficacy experiment) }\end{array}$ & Yes & Yes & Yes & Yes \\
\hline 11 & $\begin{array}{l}\text { Oumbouke et al. } \\
{[16]}\end{array}$ & Yes & Yes & Yes & Yes & Yes & Yes & Yes & Unknown & Yes & Yes & Yes & Yes \\
\hline 12 & $\begin{array}{l}\text { Snetselaar et al. } \\
{[13]}\end{array}$ & Yes & Yes & Yes & Yes & Yes & Yes & Yes & $\begin{array}{l}\text { At least four replicates } \\
\text { of treatment }\end{array}$ & Yes & Yes & Yes & Yes \\
\hline 13 & $\begin{array}{l}\text { Sternberg et al. } \\
{[14]}\end{array}$ & Yes & Yes & Yes & Yes & Yes & Yes & Yes & $\begin{array}{l}\text { At least three replicates } \\
\text { of treatment }\end{array}$ & Yes & Yes & Yes & Yes \\
\hline
\end{tabular}

Case-control (articles 1-4; 8-13)

$\# 1$. Did the study address a clearly focused issue?

\#2. Did the authors use an appropriate method to answer their question?

\#3. Were the cases recruited in an acceptable way?

\#4. Were the controls selected in an acceptable way?

\#5. Was the exposure accurately measured to minimise bias?

\#6a. Aside from the experimental intervention, were the groups treated equally?

\#6b. Have the authors taken account of the potential confounding factors in the design and/or in their analysis?

\#7. How large was the treatment effect?

\#8. How precise was the estimate of the treatment effect?

\#9. Do you believe the results?

\#10. Can the results be applied to the local population?

\#11. Do the results of this study fit with other available evidence?

Randomized controlled trial (articles 5-7):

\#1. Did the study address a clearly focused research question?

\#2. Was the assignment of participants to interventions randomised?

\#3. Were all participants who entered the study accounted for at its conclusion?

\#4. Were the participants 'blind' to the intervention they were given? Were the investigators 'blind' to the intervention they were giving to participants? Were the 
Table.2 (continued)

people assessing/analysing outcome/s'blinded'?

\#5. Were the study groups similar at the start of the randomised controlled trial?

\#6. Apart from the experimental intervention, did each study group receive the same level of care (that is, were they treated equally)?

\#7. Were the effects of intervention reported comprehensively?

\#8. Was the precision of the estimate of the intervention or treatment effect reported?

\#9. Do the benefits of the experimental intervention outweigh the harms and costs?

\#10. Can the results be applied to your local population/in your context?

\#11. Would the experimental intervention provide greater value to the people in your care than any of the existing interventions?

The black shaded areas for these articles indicate the absence of an additional critical appraisal question

interventions utilized the In2Care insert $(\mathrm{n}=2)$, transfluthrin $(\mathrm{n}=2)$, beta-cyfluthrin $(\mathrm{n}=1)$, bendiocarb $(\mathrm{n}=1)$, and pirimiphos-methyl $(\mathrm{PM})$ insecticides $(\mathrm{n}=1)$. Barreaux et al. [12] found that the In2Care tool differentially killed $25 \%$ more mosquitoes per day compared to the control hut in which $2-4 \%$ daily deaths occurred.

In another report, 11 insecticides were tested for their toxicity, wherein beta-cyfluthrin remained the most durable and potent of them all [16]. Beta-cyfluthrin was observed to maintain $100 \%$ mortality rates up to one month of use [16]. This team found that the insecticide killed $55 \%$ of mosquitoes that came into contact with treated eaves (an average of $44 \%$ of released mosquitoes) by the following morning; then, after $24 \mathrm{~h}$ of exposure, $64 \%$ of mosquitoes died, although not at a significant difference $(p>0.05)$. Within the untreated control hut, less than $5 \%$ of mosquitoes died that came into contact with untreated eaves [16].

Along with beta-cyfluthrin eaves, two interventions focused on mosquito mortality from transfluthrintreated eaves. Eaves covered with transfluthrin were reported to result in high mortality rates. In one report, around $99.5 \%$ of all mosquitoes died upon exposure to houses protected by treated eaves [10]. Moreover, Mwanga et al. [11] found that, after exposing caged mosquitoes to treated eave ribbons, all mosquitoes reportedly died.

Chinula et al. [17] investigated the wash resistance of window screens and eave baffles (WSEBs) treated with pirimiphos-methyl (PM) insecticide and polyacrylatebinding agents (BA). Exposed mosquitoes died at rates equal to that of LLIN and continued to die at $94 \%$ after 20 washes $(95 \%$ CI 0.74-0.98; p < 0.001) [17]. When BA was removed, all mosquitoes died after zero washes; however, after 10 washes, less than $10 \%$ of all mosquitoes died [17]. Likewise, when the PM insecticide was removed, less than $5 \%$ of mosquitoes died after $0,5,10$, and 20 washes whether or not BA was present [17].

\section{Participant needs and attitudes}

Only two articles discussed volunteer attitudes toward treated eaves or window screens (Table 4). Mmbando et al. [18] reported that participants had overall positive outlooks toward prototype windows because of their ventilation and attraction. A positive regard for these devices was demonstrated over 10 weeks by the doubling of male residents from four to eight in houses newly installed with prototype windows. Eventually, new inhabitants displayed housing comfort, redecorating 10 out of the 20 housing units with new curtains and floors [18]. Relative to bed nets, which can generally accommodate two people, eave ribbons treated with transfluthrin could accommodate four residents [10].

\section{Discussion}

The evidence reviewed suggests that eave tubes infused with specific insecticides can reduce vector-biting incidence rates, mosquito entry rates and mosquito prevalence (Tables 3-4). Based on the studies reviewed here, insecticide-treated eaves coated with beta-cyfluthrin or transfluthrin can reduce vector biting by noteworthy amounts: A 64\% drop in An. gambiae s.l. blood-feeding when treated with $10 \%$ beta-cyfluthrin; and a 100\% drop in indoor and outdoor biting when treated with $0.2 \%$ transfluthrin $[10,19]$. Transfluthrin appears to be one of the more effective insecticides on eave tubes for reducing mosquito biting, as at a concentration of $0.02 \%$ biting protection rates were $77.2 \%$ indoors and $56.2 \%$ outdoors [10]. Worth noting, however, is that transfluthrin-eaves appear to offer minimal to no protection against $A n$. funestus mosquitoes [10]. Public health practitioners should therefore base the type of insecticide they treat eaves with on the most prevalent species of mosquito in a community.

Households fitted with beta-cyfluthrin-treated eaves might also be protected against mosquito entry. Of all the tests included with this insecticide, the one that followed the Lethal House Lure approach was the most successful. Using this system where beta-cyfluthrin-coated eaves were installed in houses, only $0-0.4 \%$ of mosquitoes entered these structures [12]. Local governments 


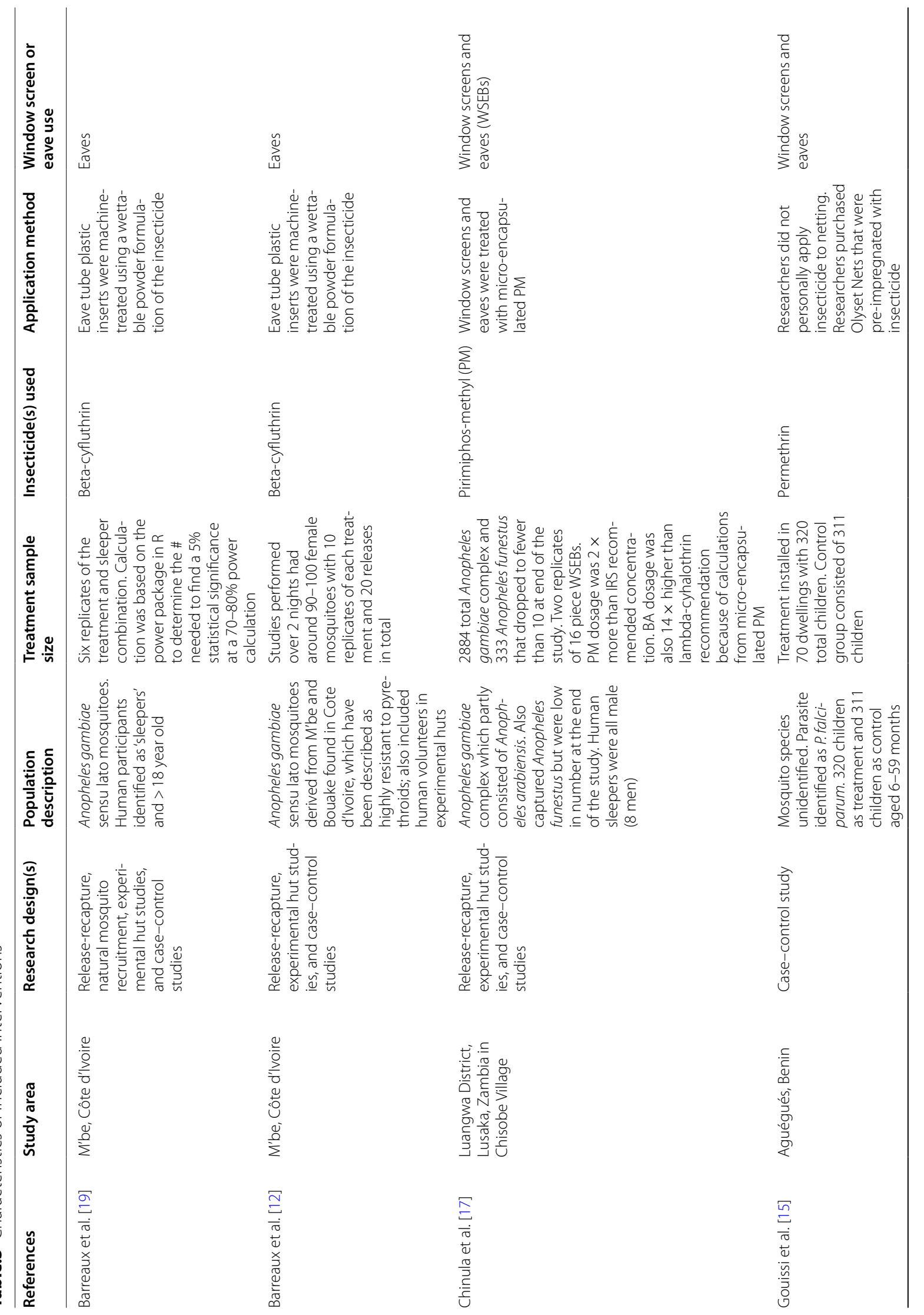




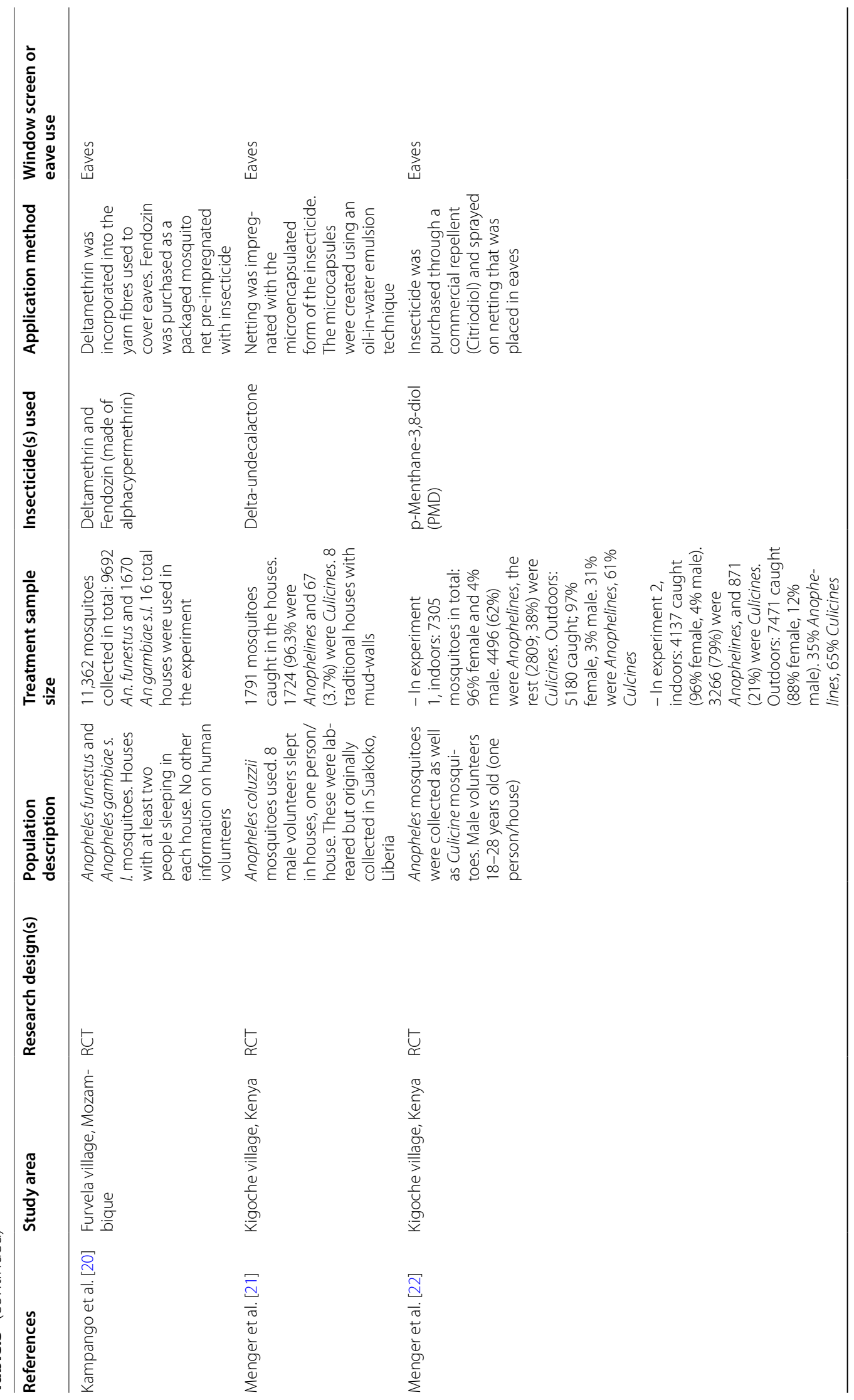




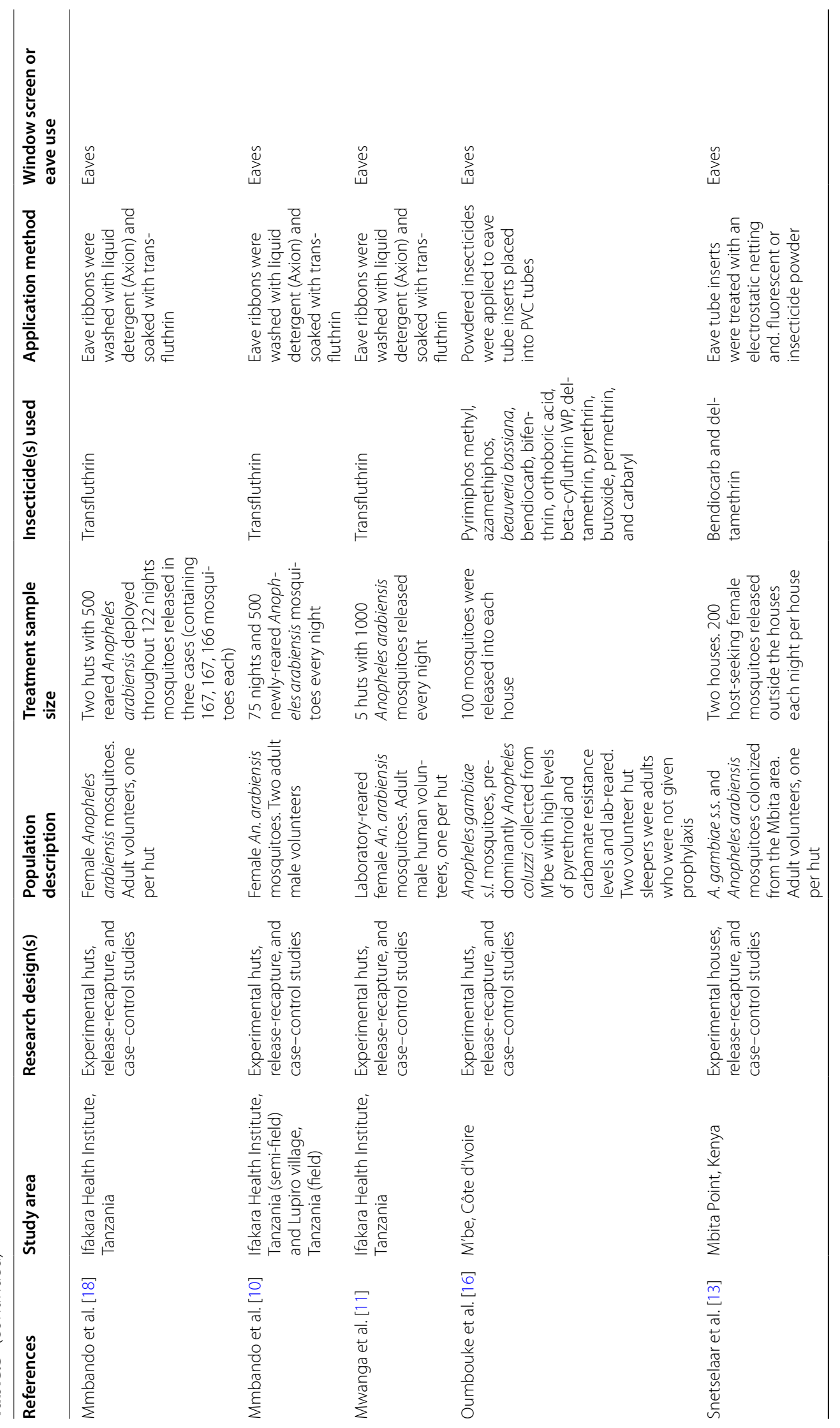




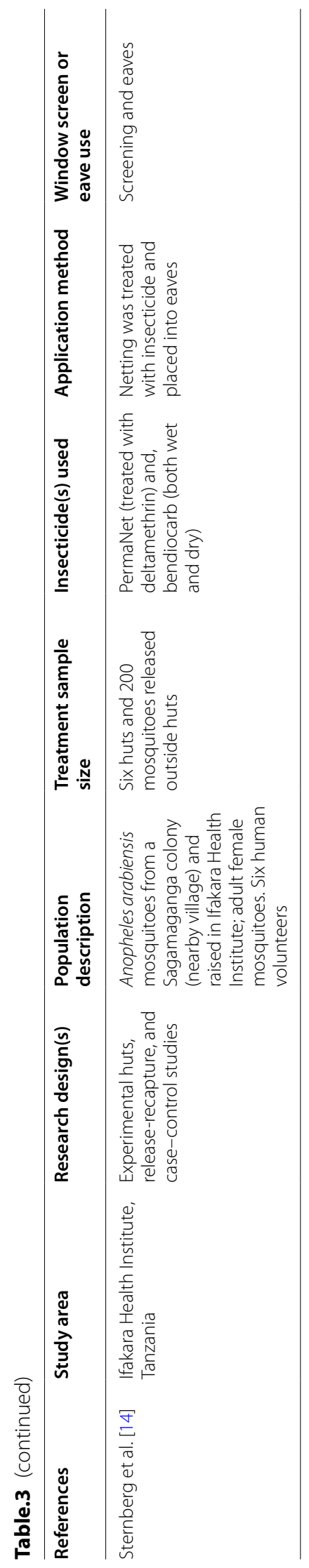




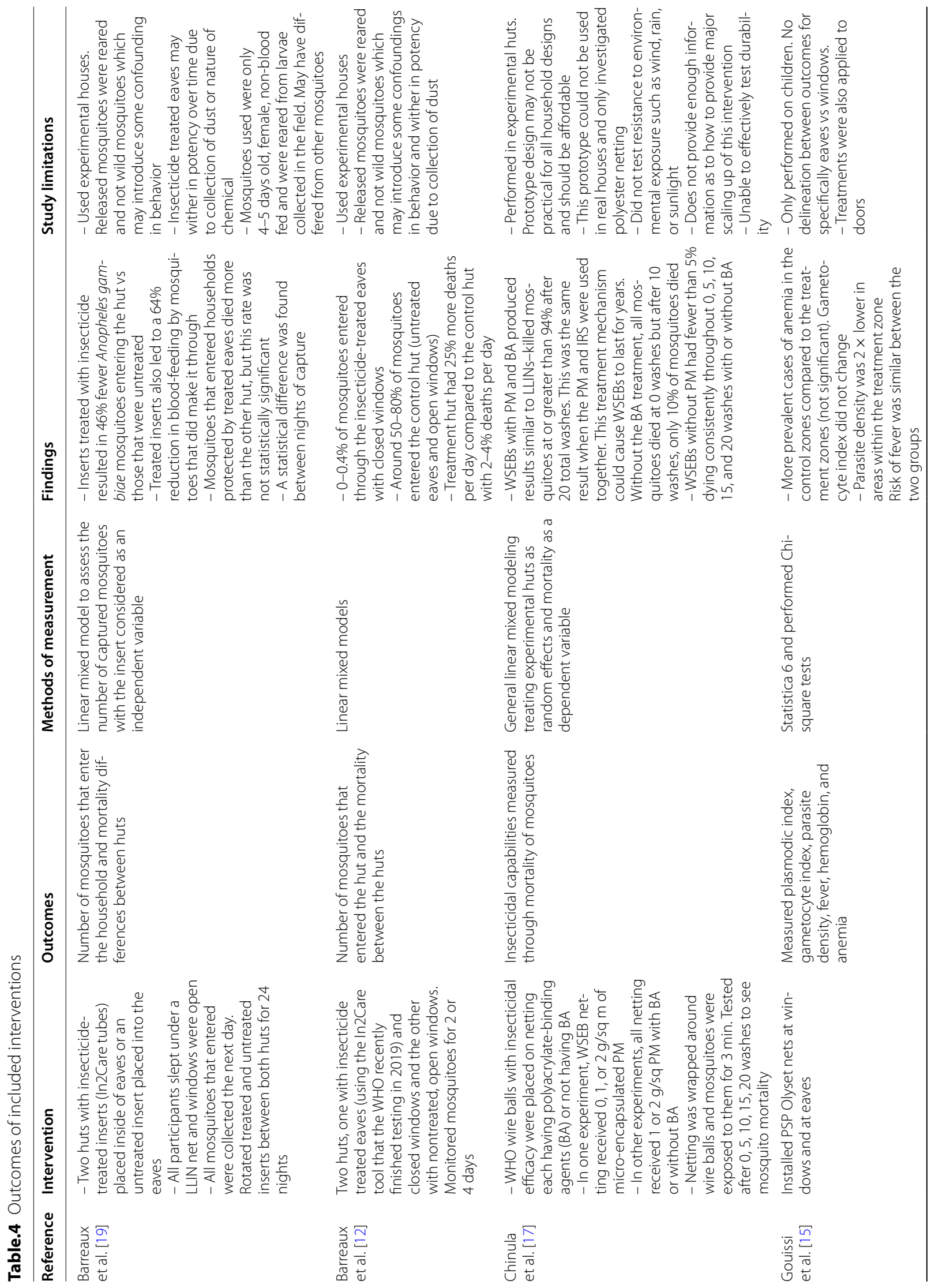




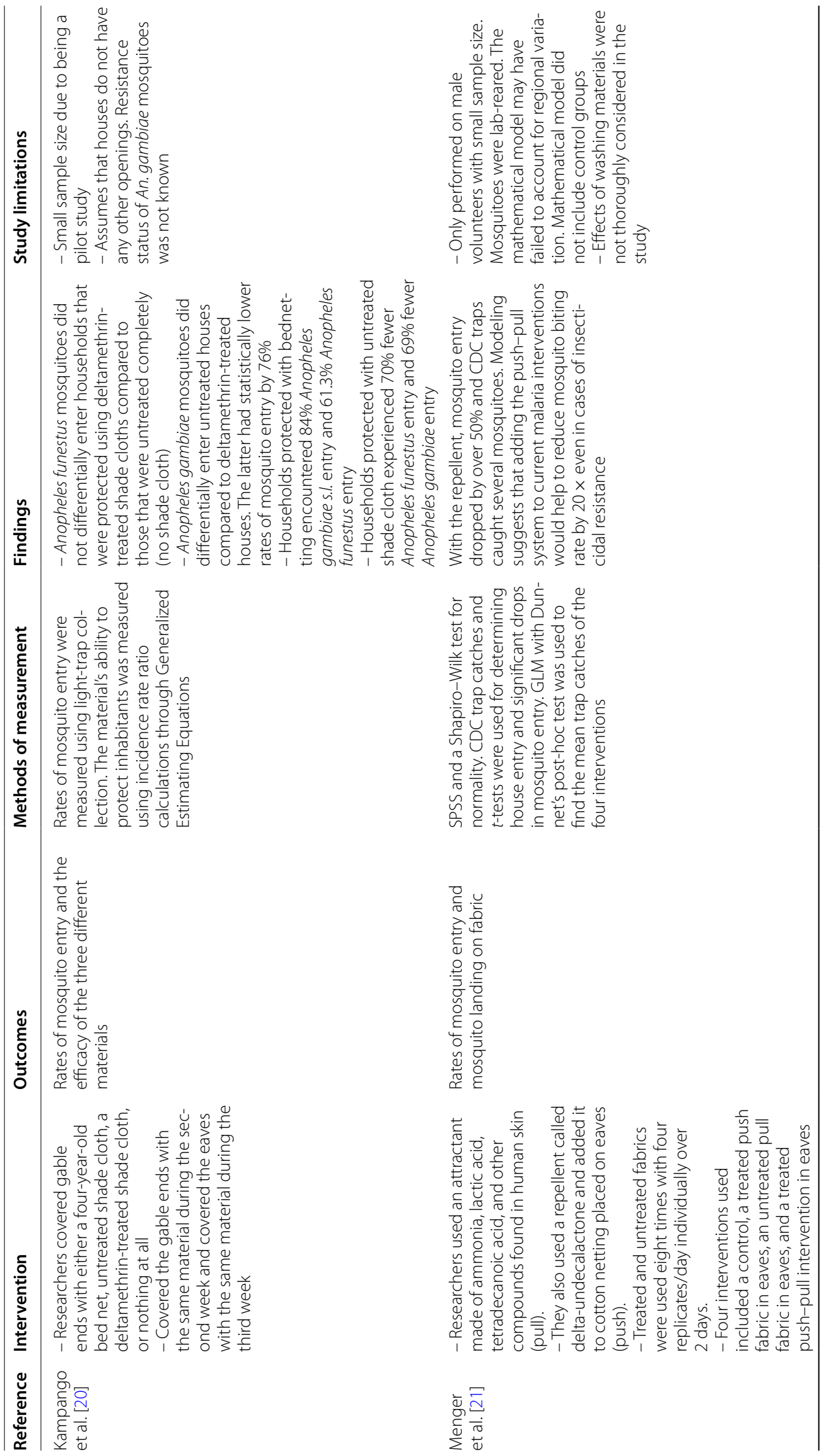



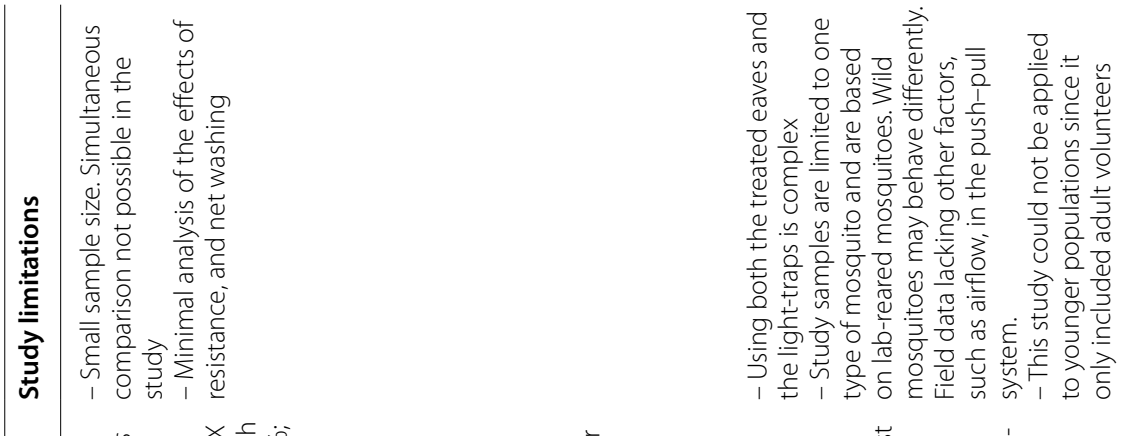

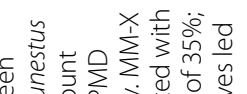

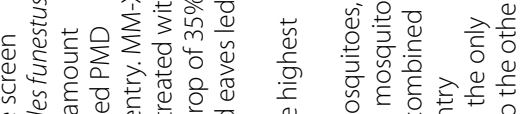

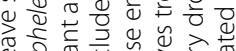

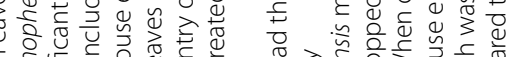

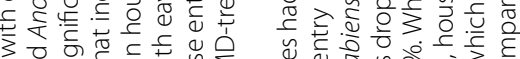

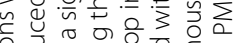

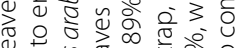

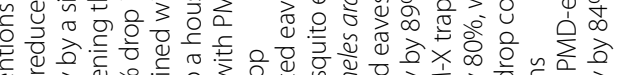

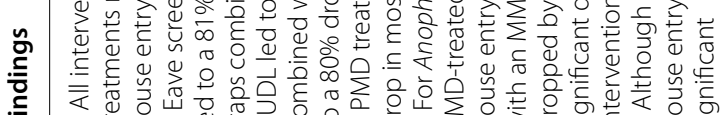

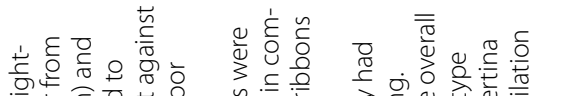

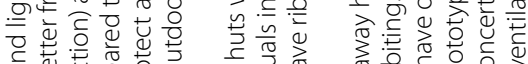

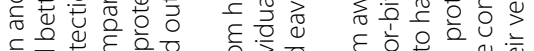

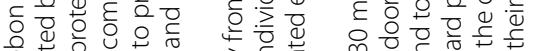

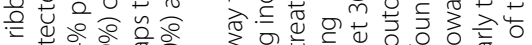

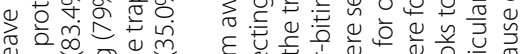

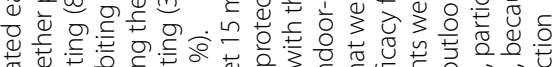

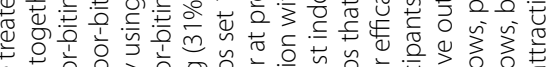

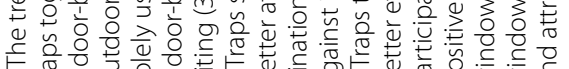
।
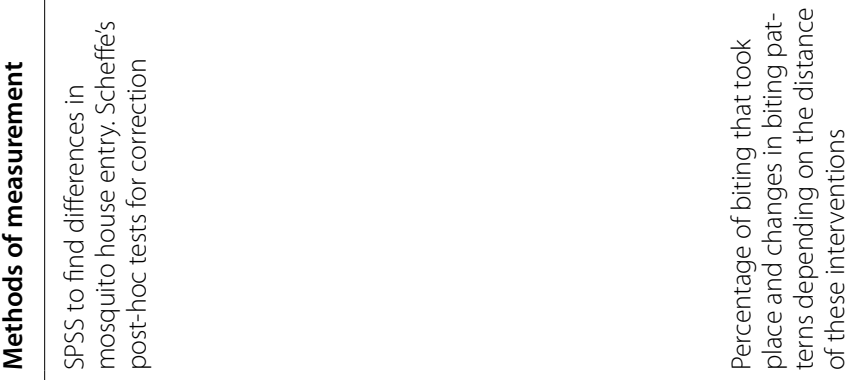

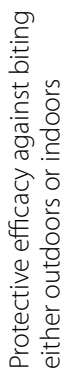

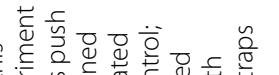

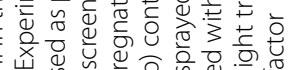

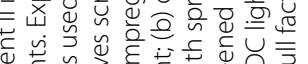

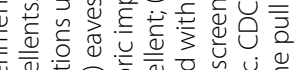

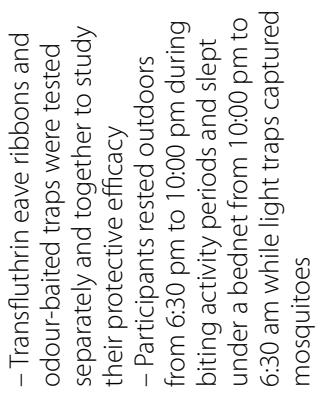

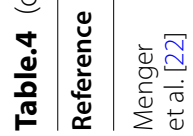

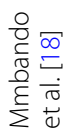




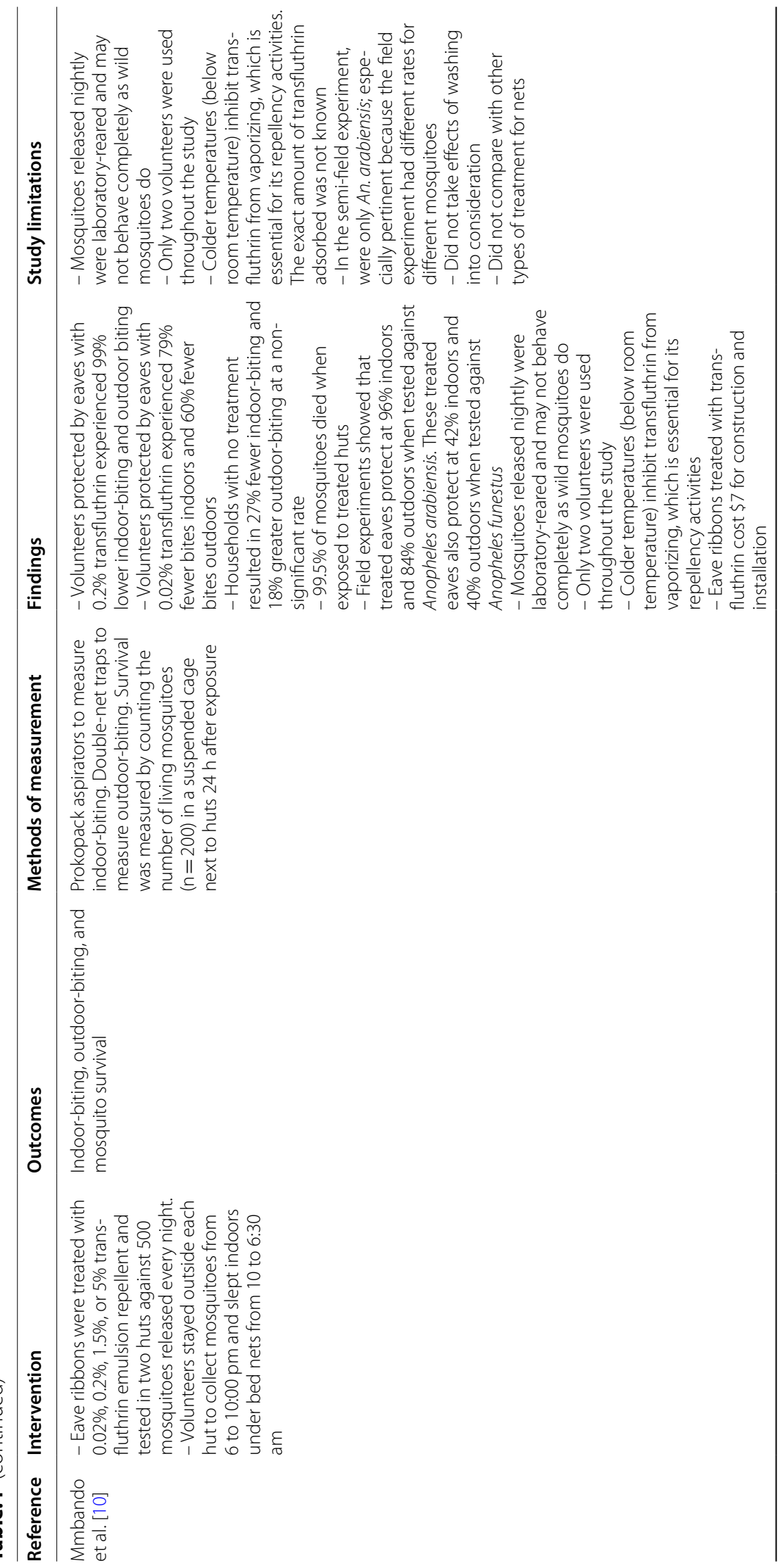



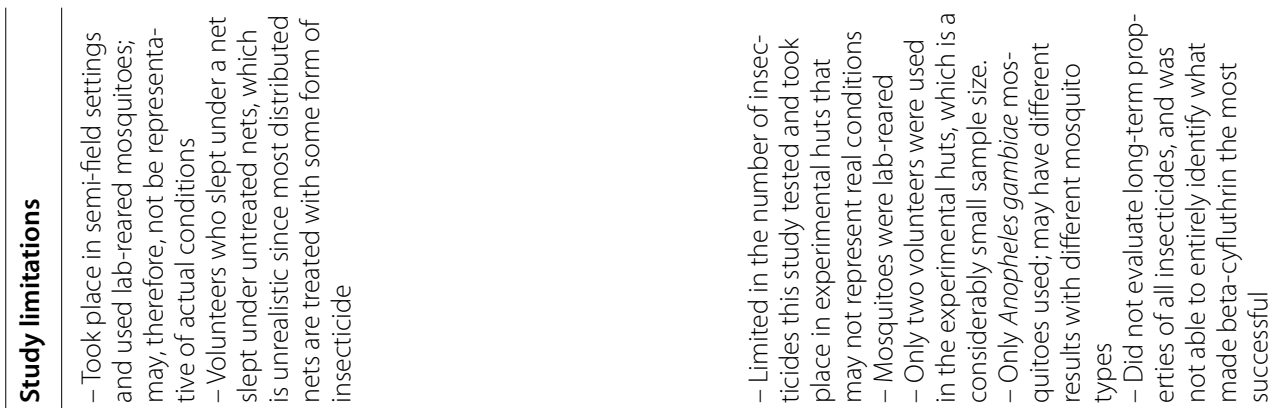

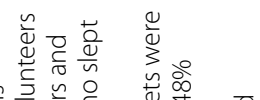

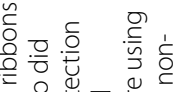

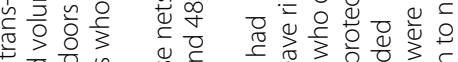

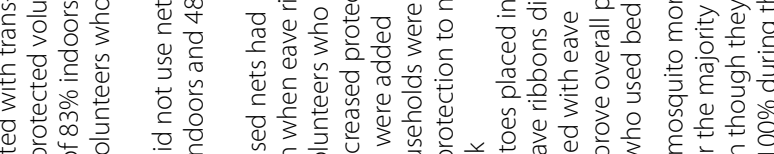

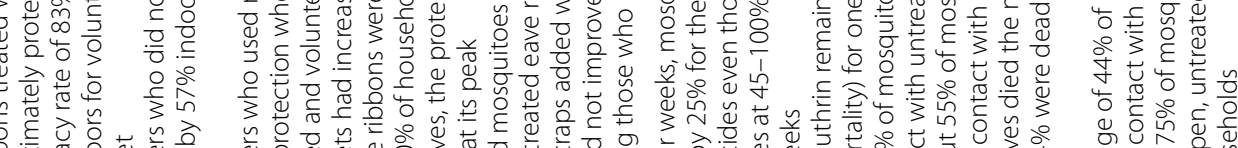

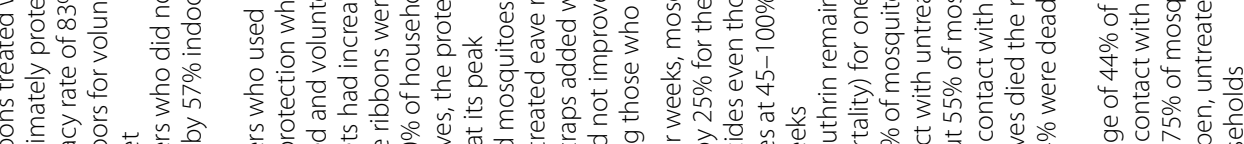

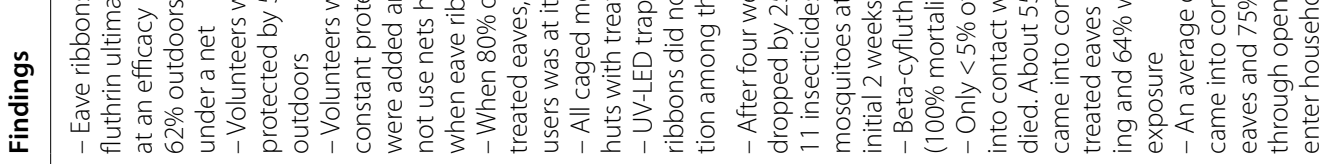

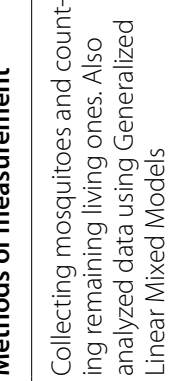

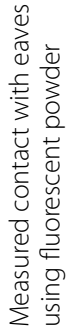

$\subseteq$

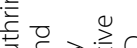

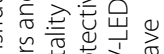

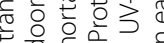

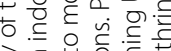

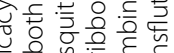

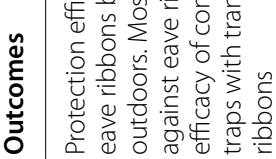

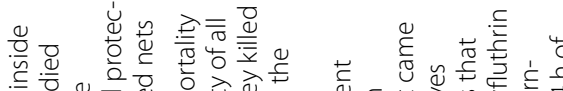

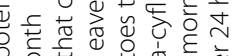

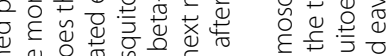

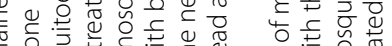

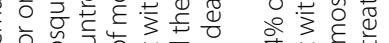




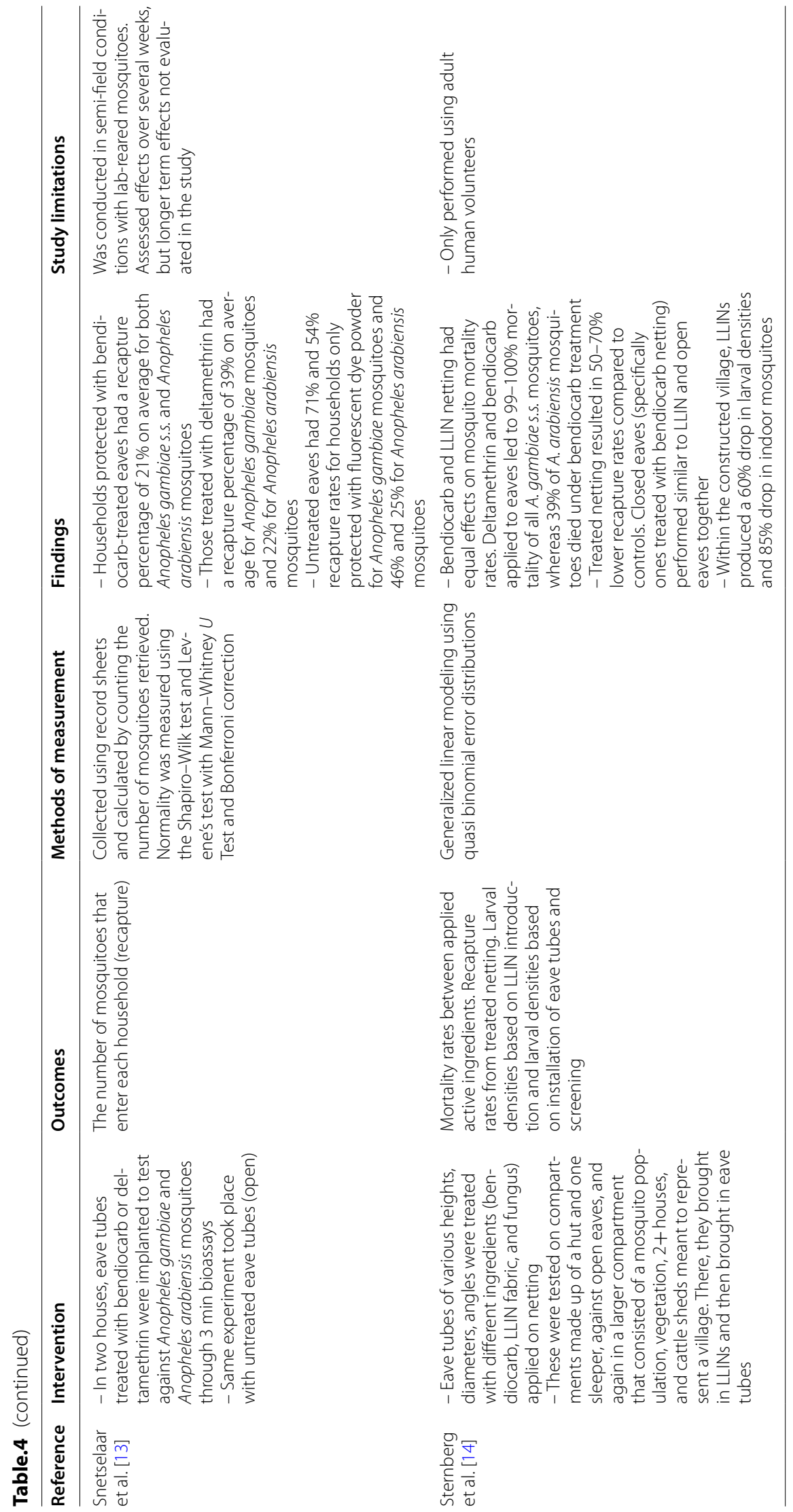


in malaria-endemic areas could consider implementing similar designs in their communities. Alternatively, applying bendiocarb to eaves resulted in a $79 \%$ drop in mosquito entry rates and could be used as a substitute for beta-cyfluthrin [13]. Alone, bendiocarb-eaves performed as well as LLIN coupled with untreated eaves, suggesting that together this intervention and LLIN may bolster preventative efforts [14].

Insecticide-treated eaves may also increase mosquito mortality. Transfluthrin was found to kill around 99.5\% of mosquitoes in one report [10], while beta-cyfluthrin treatments killed $100 \%$ of mosquitoes for at least one month in another [16]. As impressive as most of these figures are, few studies investigated the long-term use of their interventions. This is concerning because durability is an important factor in cost-benefit analyses, raising logistical questions about replacing tools that have lost their effectiveness. The compounds in an insecticide are of varying levels of photosensitivity, meaning that exposure to sunlight initiates chemical breakdown [23]. For window screens coated with insecticide, the risk of sunlight exposure is high, eventually reducing insecticidal strength over time. Eaves, on the other hand, are situated in an enclosed space and experience less sunlight exposure. Thus, the insecticidal strength of treated eaves may last longer than on window screens.

Available evidence suggests that both window screens and eaves should be co-treated with BA to retain insecticidal potency after washes [16]. The data comes specifically from research done with PM insecticide and may not translate as accurately to other insecticides. Only two of the 13 included articles discussed participant attitudes toward any of these interventions, and both of these articles were from the same research team $[10,18]$. The international community needs more data on community preferences for these tools in order that they are maintained when installed.

\section{Study strengths and limitations}

The strengths of this review include its specificity and current analysis of treated eaves and window screens against Anopheles mosquitoes. Moreover, it provides comparisons between insecticides, detailing their effectiveness and, in many cases, against what species of mosquito. This information is critical for public health researchers or programmers aiming to install said devices in affected communities, as it will help them to tailor their interventions. In addition, the research team conducted this review during a time when malaria progression expressed stagnation, thereby enhancing this review's timeliness by providing supplementary solutions [24]. The results of this study are consistent with other published reports that indicated reductions in mosquito density and biting due to eave closures or tube installations $[25,26]$.

Although specificity could be regarded as a strength, it may also be a limitation. Studies or corresponding results that tested netting without insecticide treatments, tested insecticidal resistance, or tested entomopathogenic fungi alone did not appear in this review. Similarly, many articles may have been overlooked due to the assessment of three databases. Only two reviewers, moreover, screened each paper for eligibility, which opens up this analysis to potential biases. The majority of studies were of a semi-field or entomological nature, and may not have similar results in real-life settings. Finally, none of the included articles explicitly discussed blinding of the investigators during their experiments [10-22].

\section{Conclusions}

The implementation of insecticide-treated window screens and eaves could serve as useful tools to reduce mosquito biting incidence, mosquito entry, and increase mosquito mortality (Table 4). This rapid scoping review ties into the essential public health service of developing policy, as the review is a call to action for stakeholders, such as WHO, to encourage window screen and eave installations, as well as local public health agencies to support these efforts [27]. Specific insecticides that public health practitioners can consider include transfluthrin, beta-cyfluthrin, bendiocarb, and deltamethrin, although the method and community one works with should reflect that of the associated study design. Future research should more deeply study the sole impact of treated window screen installations, interventions for An. funestus and An. arabiensis, and household attitudes toward these devices.

\footnotetext{
Abbreviations

WHO: World Health Organization; SES: Socioeconomic status; RCT: Randomized controlled trial; LLIN: Long-lasting insecticidal netting; WSEB: Window screens and eave baffles; PM: Pirimiphos-methyl; BA: Polyacrylate-binding agents; CASP: Critical appraisal skills programme checklist; PRISMA: Preferred reporting items for systematic reviews and meta-analyses.
}

\section{Acknowledgements}

The authors would like to thank Dr Paul Hunter for his invaluable help in constructing sound research strategies.

\section{Authors' contributions}

BIA and KV collected and analysed the data; BIA led the manuscript writing with substantial contributions from RF and KV; UFSO reviewed and corrected language and data interpretation. All authors read and approved the final manuscript

\section{Funding}

Funding publication made possible in part by support from the Thomas Jefferson University Open Access Fund and the College of Population Health. 


\section{Availability of data and materials}

All data generated or analysed during this study are included in this published article and its supplementary information files.

\section{Declarations}

\section{Ethics approval and consent to participate}

Not applicable.

\section{Consent for publication}

All authors reviewed and consented this manuscript's publication.

\section{Competing interests}

The authors have no conflict of interest to report. The following manuscript has been read and approved by all authors listed above. All authors have met the requirements for authorship and each believes that the manuscript represents honest work.

\section{Author details}

${ }^{1}$ College of Population Health, Thomas Jefferson University, Philadelphia, PA, USA. ${ }^{2}$ School of Medicine, Deakin University, Geelong, VIC, Australia. ${ }^{3}$ South East Zonal Biotechnology Centre and Department of Zoology and Environmental Biology, University of Nigeria, Nsukka, Nigeria.

Received: 20 May 2021 Accepted: 17 September 2021

Published online: 29 September 2021

\section{References}

1. WHO. World malaria report 2020. Geneva, World Health Organization. 2020. https://www.who.int/teams/global-malaria-programme/reports/ world-malaria-report-2020\#: :text=The\%202020\%20edition\%20of\% 20the\%20World\%20malaria\%20report, 1.5\%20billion\%20cases\%20and\% 207.6\%20million\%20deaths\%20averted. Accessed 7 July 2020.

2. Tusting LS, Ippolito MM, Willey BA, Kleinschmidt I, Dorsey G, Gosling RD, et al. The evidence for improving housing to reduce malaria: a systematic review and meta-analysis. Malar J. 2015;14:209.

3. Tusting LS, Rek J, Arinaitwe E, Staedke SG, Kamya MR, Cano J, et al. Why is malaria associated with poverty? Findings from a cohort study in rural Uganda. Infect Dis Poverty. 2016;5:78.

4. Tusting LS, Gething PW, Gibson HS, Greenwood B, Knudsen J, Lindsay SW, et al. Housing and child health in sub-Saharan Africa: a cross-sectiona analysis. PLoS Med. 2020;17: e1003055.

5. Tusting LS, Bottomley C, Gibson H, Kleinschmidt I, Tatem AJ, Lindsay SW, et al. Housing improvements and malaria risk in sub-Saharan Africa: a multi-country analysis of survey data. PLoS Med. 2017;14: e1002234.

6. Ogada J. Prioritise window screening to control malaria in Africa. 2019. https://www.scidev.net/sub-saharan-africa/news/prioritise-window-scree ning-to-control-malaria-in-africa/. Accessed 9 May 2021.

7. Moher D, Liberati A, Tetzlaff J, Altman DG, PRISMA Group. Preferred reporting items for systematic reviews and meta-analyses: the PRISMA statement. PLoS Med. 2009;6: e1000097.

8. Critical Appraisal Checklist Skills Programme. CASP checklist. 2018. https://casp-uk.b-cdn.net/wp-content/uploads/2018/03/CASP-CaseControl-Study-Checklist-2018_fillable_form.pdf. Accessed 25 July 2021.

9. Critical Appraisal Checklist Skills Programme. CASP randomised controlled trial standard checklist. 2020. https://casp-uk.b-cdn.net/wp-conte nt/uploads/2020/10/CASP_RCT_Checklist_PDF_Fillable_Form.pdf. Accessed 25 July 2021.

10. Mmbando AS, Ngowo H, Limwagu A, Kilalangongono M, Kifungo K, Okumu FO. Eave ribbons treated with the spatial repellent, transfluthrin, can effectively protect against indoor-biting and outdoor-biting malaria mosquitoes. Malar J. 2018;17:368.

11. Mwanga EP, Mmbando AS, Mrosso PC, Stica C, Mapua SA, Finda MF, et al. Eave ribbons treated with transfluthrin can protect both users and nonusers against malaria vectors. Malar J. 2019;18:314.
12. Barreaux AMG, Oumbouke WA, Tia IZ, Brou N, Koffi AA, N'guessan R, et al. Semi-field evaluation of the cumulative effects of a "lethal house lure" on malaria mosquito mortality. Malar J. 2019;18:298.

13. Snetselaar J, Njiru BN, Gachie B, Owigo P, Andriessen R, Glunt K, et al. Eave tubes for malaria control in Africa: prototyping and evaluation against Anopheles gambiae s.s. and Anopheles arabiensis under semi-field conditions in western Kenya. Malar J. 2017;16:276

14. Sternberg ED, Ng'habi KR, Lyimo IN, Kessy ST, Farenhorst M, Thomas MB, et al. Eave tubes for malaria control in Africa: initial development and semi-field evaluations in Tanzania. Malar J. 2016;15:447.

15. Gouissi FM, Salifou S, Edorh AP, Sedjame AR, Gouissi SGA, Yadouleton WA, et al. Contribution of poses screen preimpregnated (PSP) installed at openings and eaves of dwellings in the reduction of malaria transmission in the commune of Aguégués in Bénin. Asian Pacific J Trop Med. 2013;6:61-7.

16. Oumbouke WA, Tia IZ, Barreaux AMG, Koffi AA, Sternberg ED, Thomas MB, et al. Screening and field performance of powder-formulated insecticides on eave tube inserts against pyrethroid resistant Anopheles gambiae s.l. an investigation into 'actives' prior to a randomized controlled trial in Côte d'Ivoire. Malar J. 2018;17:374.

17. Chinula D, Sikaala CH, Chanda-Kapata P, Hamainza B, Zulu R, Reimer L, et al. Wash-resistance of pirimiphos-methyl insecticide treatments of window screens and eave baffles for killing indoor-feeding malaria vector mosquitoes: an experimental hut trial, south east of Zambia. Malar J. 2018:17:164.

18. Mmbando AS, Batista EPA, Kilalangongono M, Finda MF, Mwanga EP, Kaindoa EW, et al. Evaluation of a push-pull system consisting of transfluthrintreated eave ribbons and odour-baited traps for control of indoor-and outdoor-biting malaria vectors. Malar J. 2019;18:87.

19. Barreaux AMG, Brou N, Koffi AA, N'Guessan R, Oumbouke WA, Tia IZ, et al. Semi-field studies to better understand the impact of eave tubes on mosquito mortality and behaviour. Malar J. 2018;17:306.

20. Kampango A, Bragança M, de Sousa B, Charlwood JD. Netting barriers to prevent mosquito entry into houses in southern Mozambique: a pilot study. Malar J. 2013:12:99.

21. Menger DJ, Omusula P, Holdinga M, Homan T, Carreira AS, Vandendaele P, et al. Field evaluation of a push-pull system to reduce malaria transmission. PLoS One. 2015;10: e0123415.

22. Menger DJ, Omusula P, Wouters K, Oketch C, Carreira AS, Durka M, et al. Eave screening and push-pull tactics to reduce house entry by vectors of malaria. Am J Trop Med Hyg. 2016;94:868-78.

23. Cavallaro MC. Assessing the effects of chronic neonicotinoid insecticide exposure on aquatic insects using multiple experimental approaches. CORE. 2018. https://core.ac.uk/download/pdf/226115425.pdf. Accessed 25 July 2021.

24. WHO. World malaria report 2019. Geneva, World Health Organization. 2019. https://www.who.int/news-room/feature-stories/detail/worldmalaria-report-2019. Accessed 7 July 2020.

25. Rek JC, Alegana V, Arinaitwe E, Cameron E, Kamya MR, Katureebe A, et al. Rapid improvements to rural Ugandan housing and their association with malaria from intense to reduced transmission: a cohort study. Lancet Planet Health. 2018;2:e83-94.

26. Sternberg ED, Cook J, Alou LPA, Assi SB, Koffi AA, Doudou DT, et al. Impact and cost-effectiveness of a lethal house lure against malaria transmission in central Côte d'Ivoire: a two-arm, cluster-randomised controlled trial. Lancet. 2021;397:805-15.

27. Centers for Disease Control and Prevention. 10 essential public health services. 2020. https://www.cdc.gov/publichealthgateway/publicheal thservices/essentialhealthservices.html. Accessed 7 July 2020.

\section{Publisher's Note}

Springer Nature remains neutral with regard to jurisdictional claims in published maps and institutional affiliations. 